\title{
HPTLC Phenolic Profiles as Useful Tools for the Authentication of Honey
}

\author{
Natalia Stanek ${ }^{1}$ • Izabela Jasicka-Misiak ${ }^{1}$ \\ Received: 22 December 2017 / Accepted: 29 April 2018 / Published online: 7 May 2018 \\ (C) The Author(s) 2018
}

\begin{abstract}
The present study reveals the utility of high-performance thin-layer chromatographic (HPTLC) fingerprinting of phenolic constituents for the authentication of monofloral honeys. The obtained data enables a more complete assessment of honey quality and the identification of emerging threats to honey quality. The developed procedure facilitates differentiation of varietal honeys and detection of honey adulterations. We used an HPTLC fingerprint analysis to determine the characteristic patterns of different honey types (willow, buckwheat, heather, pine honeydew, and manuka honey). The HPTLC chromatograms were used to determine the differences in the botanical origin of the honey samples on the basis of the band profiles, which are characteristic for each honey type. Identification of 11 polyphenols was performed by comparison of the color and $R_{f}$ of the bands with available standards. Additionally, the results were confirmed by an HPLC analysis.
\end{abstract}

Keywords Honey $\cdot$ Phenolic compounds $\cdot$ HPTLC fingerprint $\cdot$ High-performance thin-layer chromatography

\section{Introduction}

Phenolic compounds rank among the most important compounds present in honey. They exhibit many biological effects, including their actions as antioxidant, antibacterial, anti-inflammatory, and anti-allergic agents. Therefore, honey, despite being considered a highly nutritious natural product, is also used for the prevention and treatment of many diseases. In recent decades, different methods have been implemented for the authentication of honey (Karabagias et al. 2014, Dinca et al. 2015, Ciulu et al. 2016, Pascual-Maté et al. 2017), and the analysis of phenolic compounds is well documented as a very promising method for determining the floral and geographical origins of honey. In general, an analytical procedure for the determination of phenolic compounds includes three steps: isolation, separation and identification, and the most common method of separation is high-performance liquid chromatography (HPLC) or capillary electrophoresis (CE) (Pyrzyńska and Biesaga 2009). In addition, statistical

Izabela Jasicka-Misiak

izajm@uni.opole.pl

Natalia Stanek

nstanek@uni.opole.pl

1 Faculty of Chemistry, Opole University, Oleska 48, 45-052 Opole, Poland methods, such as hierarchical cluster analysis, principal component analysis, or discriminant analysis are increasingly introduced for the study of the usefulness of different analytical technique used to authenticate honey (Kečkeš et al. 2013; Kuś et al. 2014; Kasprzyk et al. 2018).

This study presents the determination of the phenolic profiles of various honey samples by using high-performance thin layer chromatography (HPTLC) as a detection method. Although HPLC still remains the most dominant technique for the separation and identification of phenolic compounds (Badjah Hadj Ahmed et al. 2016, Pascual-Maté et al. 2017), HPTLC seems to be a very promising alternative for the analysis of these compounds in honey (Locher et al. 2017). This is because HPTLC is simpler, more flexible, more accessible, and cheaper than other commonly used methods. Furthermore, HPTLC allows a parallel separation and quantitative determination of many samples at the same time (Ristivojević et al. 2017). HPTLC chromatograms can be used to determine the differences in botanical origins of honey samples by exhibiting a characteristic set of common bands that is typical for each analyzed branch. Thus, the entire chromatogram is treated as a unique, distinctive fingerprint for each selected type of honey. Chromatograms could, thus, complement quality-control tools for honey. An equally important feature of the HPTLC analysis of phenolic compounds is the detection of individual chemical species that are present in certain honey. Separated substances can be detected by determining the color of the substance, the fluorescence of 
the substance in UV light, or the color reactions of separated substances. Individual classes of phenolic compounds exhibit different spot coloration under UV light with visualizing reagents. Specifically, flavonoids, like quercetin, show orange or yellow spots, while flavones appear dull brown. Anthocyanins are detected as purple-red-orange or sometimes yellow bands in visible light. Light and dark blue spots are characteristic for caffeic, $p$-coumaric, and ferulic acids. On the other hand, simple phenolics, such as gallic or salicylic acids, do not fluoresce under UV light and quench to give dark absorbing spots (Waterman 1994). Thus, Głowniak et al. 2005 analyzed phenolic compounds in the flowers of Lavatera trimestris $L$. The fluorescence colors of phenolic acid and flavonoid standards were blue or light blue for caffeic, $p$-coumaric, ferulic, and chlorogenic acids. Additionally, caffeic, ferulic, and chlorogenic acids, after spraying with a $2 \%$ aqueous solution of ferric chloride, produced brown-green red, light-orange, and gray-green spots, respectively. Milojković Opsenica et al. 2016 analyzed phenolic compounds in propolis samples. Propolis of so-called Otype showed orange (typical for flavonoids), light blue, and some green bands. Orange, green-brown, light blue, and green bands were further identified as quercetin, chrysin, caffeic acid, and naringenin, respectively. It is noteworthy that only one paper has been devoted to the analysis of the chemical composition of honey by means of HPTLC. Locher et al. 2017 explored HPTLC fingerprinting for the authentication of honey, such as jarrah (Eucalyptus marginata), marri (Corymbia calophyla), and manuka (Leptospermum scoparium), but they did not study the use of the HPTLC method for the determination of phenolic compounds.

The aim of the present work was fingerprinting of phenolics by HPTLC to determine their characteristic patterns in willow, buckwheat, heather, pine honeydew, and manuka honey.

\section{Materials and Methods}

\section{Honey Samples}

Seventeen honey samples of five different varieties of honey (willow, heather, buckwheat, pine honeydew, and manuka) were analyzed. The types of pollen present in honeys analyzed are shown in Table 1. Two categories of honey were distinguished: pure honeys (W2-W3, H1-H2, B1, M1-M2) and honeys with pollen content slightly lower than $45 \%$ (W1, B2-B4). In the case of pine honeydew honeys, the authentication is not carried out on the basis of the pollen grains, and their classifications is made by using electrical conductivity parameter. According to Polish Quality Standard (PN-88/A77626 1998) and Bogdanov et al. 2004, the electrical conductivity should be higher than $800 \mu \mathrm{S} / \mathrm{cm}$, then honey is a honeydew.
The all honeydew honey samples had an electrical conductivity in the range of $1160-1580 \mu \mathrm{S} / \mathrm{cm}$. The honey samples were of different harvesting years (2015-2016) and geographical origins (Poland, New Zealand, and Australia) (Table 1). The products were stored at $4{ }^{\circ} \mathrm{C}$ in the dark.

\section{Chemicals and Reagents}

All chemicals and solvents used for the chromatography column, HPLC and HPTLC analyses were of analytical purity grade. Methanol, ethanol dibasic sodium phosphate heptahydrate $\left(\mathrm{Na}_{2} \mathrm{HPO}_{4} \times 7 \mathrm{H}_{2} \mathrm{O}\right), 85 \%$ phosphoric acid, chloroform, ethyl acetate, and formic acid were purchased from POCH S.A. (Gliwice, Poland). The standards (chrysin, myricetin, 3,4-dihydroxybenzoic acid, caffeic acid, chlorogenic acid, ellagic acid, ferulic acid, gallic acid, $p$ coumaric acid, rosmarinic acid, and ( \pm )-abscisic acid) were purchased from Sigma-Aldrich (Poznań, Poland). Amberlite XAD-2, which was used as an adsorption resin for the extraction step, was obtained from Supelco (Bellefonte, PA, USA).

\section{Physicochemical Properties}

Selected physicochemical parameters: moisture content, $\mathrm{pH}$, and electrical conductivity have been determined using the Polish Quality Standard (PN-88/A-77626).

\section{Phenolic Extraction (Solid-Phase Extraction, SPE)}

The extraction was performed using the method described by Jasicka-Misiak et al. (2017). The honey (25 g) was thoroughly mixed with five parts $(125 \mathrm{~mL})$ of acidified water $(\mathrm{pH}=2$ with $\mathrm{HCl}$ ) and homogenized in an ultrasonic bath for $60 \mathrm{~min}$. The solutions were then filtered through filter paper to remove any solid particles. Amberlite XAD-2 (Supelco, Bellefonte, PA, USA, pore size $9 \mathrm{~nm}$, particle size $0.3-1.2 \mathrm{~mm}$ ) was packed into a glass column $(2.5 \mathrm{~cm} \times 55 \mathrm{~cm})$ and washed with both methanol and acidified water. Next, the honey extract was transferred to a glass column and kept for 30-45 min. The solid phase was washed with acidified water $(0.05 \mathrm{M} \mathrm{HCl}$, $250 \mathrm{~mL})$ and neutral distilled water $(300 \mathrm{~mL})$ to remove all sugars and other polar compounds. The polyphenolics were eluted from the sorbent with methanol $(500 \mathrm{~mL})$. The methanol extracts were evaporated under reduced pressure at $40^{\circ} \mathrm{C}$. The dried residue was then dissolved in methanol (HPLCgrade).

\section{HPTLC Separation}

The $10 \mu \mathrm{L}$ of the honey extracts and $4 \mu \mathrm{L}$ of the standard solutions (concentration of $1 \mathrm{mM}$ ) were applied as $8 \mathrm{~mm}$ bands on HPTLC sheets at rate of $150 \mathrm{~nL} \mathrm{~s}^{-1}$ using a semiautomated HPTLC application device (Linomat 5, 
Table 1 Characteristic of studied honey samples

\begin{tabular}{|c|c|c|c|c|c|c|}
\hline \multirow[t]{2}{*}{$\begin{array}{l}\text { Type of honey, } \\
\text { floral origin }\end{array}$} & \multirow[t]{2}{*}{$\begin{array}{l}\text { Place and year } \\
\text { of production }\end{array}$} & \multirow[t]{2}{*}{ Sample ID } & \multirow[t]{2}{*}{$\begin{array}{l}\text { Predominant } \\
\text { pollen type [\%] }\end{array}$} & \multicolumn{3}{|c|}{$\begin{array}{l}\text { Selected chemical parameters of honey } \\
\text { (Polish Quality Standard PN-88/A-77626) } n=3\end{array}$} \\
\hline & & & & $\begin{array}{l}\text { Moisture } \\
\text { content }[\%]\end{array}$ & $\begin{array}{l}\text { Electric conductivity } \\
{[\mathrm{mS} / \mathrm{cm}]}\end{array}$ & $\mathrm{pH}$ \\
\hline \multirow[t]{3}{*}{ Willow, Salix spp. } & Poland, 2015 & W1 & 27.0 & $16.3 \pm 2.2$ & $0.38 \pm 0.15$ & 3.98 \\
\hline & Poland, 2015 & W2 & 59.0 & $16.1 \pm 1.6$ & $0.40 \pm 0.09$ & 3.97 \\
\hline & Poland, 2016 & W3 & 65.0 & $16.4 \pm 2.1$ & $0.64 \pm 0.11$ & 3.92 \\
\hline \multirow[t]{2}{*}{ Heather, Calluna vulgaris L. } & Poland, 2016 & $\mathrm{H} 1$ & 57.4 & $18.6 \pm 1.5$ & $0.66 \pm 0.12$ & 4.34 \\
\hline & Poland, 2016 & $\mathrm{H} 2$ & 73.8 & $18.1 \pm 1.3$ & $0.64 \pm 0.08$ & 4.39 \\
\hline \multirow{4}{*}{$\begin{array}{l}\text { Buckwheat, } \\
\text { Fagopyrum esculentum L. }\end{array}$} & Poland, 2016 & B1 & 48.6 & $17.1 \pm 1.9$ & $0.43 \pm 0.09$ & 3.79 \\
\hline & Poland, 2016 & $\mathrm{~B} 2$ & 41.9 & $16.5 \pm 3.1$ & $0.46 \pm 0.10$ & 3.86 \\
\hline & Poland, 2016 & B3 & 33.9 & $16.1 \pm 1.9$ & $0.39 \pm 0.07$ & 4.02 \\
\hline & Poland, 2016 & B4 & 41.1 & $16.3 \pm 1.7$ & $0.44 \pm 0.11$ & 3.81 \\
\hline \multirow{6}{*}{$\begin{array}{l}\text { Pine honeydew, } \\
\text { Pinus sylvestris L. }\end{array}$} & Poland, 2015 & PH1 & - & $16.9 \pm 2.2$ & $1.24 \pm 0.15$ & 4.57 \\
\hline & Poland, 2015 & $\mathrm{PH} 2$ & - & $16.2 \pm 1.4$ & $1.45 \pm 0.13$ & 4.53 \\
\hline & Poland, 2015 & PH3 & - & $16.4 \pm 2.1$ & $1.29 \pm 0.20$ & 4.51 \\
\hline & Poland, 2015 & PH4 & - & $16.9 \pm 2.2$ & $1.58 \pm 0.19$ & 4.62 \\
\hline & Poland, 2015 & PH5 & - & $17.1 \pm 2.4$ & $1.21 \pm 0.16$ & 4.53 \\
\hline & Poland, 2015 & PH6 & - & $16.5 \pm 1.6$ & $1.16 \pm 0.12$ & 4.50 \\
\hline \multirow{2}{*}{$\begin{array}{l}\text { Manuka, Leptospermum } \\
\text { scoparium }\end{array}$} & New Zeland, 2015 & M1 & 76.0 & $16.8 \pm 1.5$ & $0.47 \pm 0.09$ & 4.27 \\
\hline & Australia, 2016 & M2 & 64.8 & $17.3 \pm 1.9$ & $0.54 \pm 0.12$ & 4.44 \\
\hline
\end{tabular}

CAMAG, Muttenz, Switzerland). The chromatographic separations were performed on $20 \times 10 \mathrm{~cm}(0.25$ thick $)$ TLC plates (HPTLC Sillica gel 60, Merck) using a mixture of chloroform:ethyl acetate:formic acid $(5: 4: 1)$ as the mobile phase. Visualization was performed in two ways: (1) under long-wave UV light (254 nm, $366 \mathrm{~nm}$ ) and (2) by spraying with $1 \%$ methanolic $\mathrm{AlCl}_{3}$, Ce-P-Mo spraying reagent $(10 \mathrm{~g}$ of $\mathrm{Ce}\left(\mathrm{SO}_{4}\right)_{2}$, and $20 \mathrm{~g}$ of phosphomolybdic acid in $1 \mathrm{~L}$ of $10 \%$ $\mathrm{H}_{2} \mathrm{SO}_{4}$ ) (Żyszka et al. 2017) or $1 \%$ methanolic 2-aminoethyl diphenylborate (Neu's reagent), drying, and visualizing under both $254 \mathrm{~nm}$ and $366 \mathrm{~nm}$. After spraying, the plates were dried for approximately $3 \mathrm{~min}$ in warm air until colored spots appeared. The obtained chromatographic results were analyzed using HPTLC software (visionCATS, CAMAG).

\section{HPLC Separation}

An UltiMate 3000 Dionex HPLC system was used to analyze the polyphenolic compounds in the honey samples. The chromatographic separation was achieved using a reserved-phase Gemini $5 \mu \mathrm{m} \mathrm{C}_{18}$ column $(250 \times 4.6 \mathrm{~mm}$, particle size $5 \mu \mathrm{m})$. The mobile phases were methanol (solvent $\mathrm{A}$ ) and $0.01 \mathrm{M}$ aqueous phosphate buffer at $\mathrm{pH}=2.5$ (solvent $\mathrm{B}$ ) at a flow rate of $1 \mathrm{~mL} / \mathrm{min}$ in a gradient elution mode. The gradient separation was as follows (A:B ratio): 10-90\% $t=0 ; 40$ $60 \% t=13.5 \mathrm{~min} ; 90-10 \% t=39 \mathrm{~min} ; 100-0 \% t=42 \mathrm{~min}$; $10-90 \% t=55 \mathrm{~min}$. The standards and honey extracts were injected into the system with an auto-sampler, and the phenolic compounds were detected using a photodiode array detector (PDA). Stock solutions of each standard were prepared with HPLC grade methanol at a concentration of $200 \mu \mathrm{g} / \mathrm{ml}$ and were stored at $4{ }^{\circ} \mathrm{C}$ while protecting from light.

\section{Method of Validation}

\section{Calibration and Linearity}

The calibration curves were generated using nine data points: $0.05,0.1,0.5,1,5,10,50,100$, and $200 \mu \mathrm{g} / \mathrm{ml}$. Standard solutions were prepared in HPLC grade methanol and analyzed in triplicate. Linear calibration plots were obtained over the calibration range of $0.05-200 \mu \mathrm{g} / \mathrm{ml}$. The correlation coefficient $\left(r^{2}\right)$ was found to be $>0.9972$ (Table 2) for each regression equation.

\section{Precision}

The repeatability of the sample application and measurement of the peak area was tested by injecting three replicates of the standard $(100 \mu \mathrm{g} / \mathrm{ml})$ on the same day and over 3 days (three replicates each day). The intra- and inter-day variations were achieved with relative standard deviation (RSD) values lower than $3.3 \%$ for all the analyzed compounds (Table 2). 
Table 2 Correlation coefficients, LOD, LOQ, and precision of standards

\begin{tabular}{llllll}
\hline Standards & $R^{2}$ & LOD $(\mu \mathrm{g} / \mathrm{ml})$ & LOQ $(\mu \mathrm{g} / \mathrm{ml})$ & \multicolumn{2}{l}{ Precision $(\mathrm{RSD} \%)$} \\
\cline { 5 - 6 } & & & & Inter-day & Intra-day \\
\hline Chlorogenic acid & 0.9999 & 0.010 & 0.029 & 0.6 & 3.3 \\
Gallic acid & 0.9996 & 0.016 & 0.049 & 1.8 & 1.9 \\
Caffeic acid & 0.9993 & 0.046 & 0.140 & 1.4 & 3.1 \\
Ferulic acid & 0.9994 & 0.010 & 0.032 & 0.6 & 1.0 \\
p-coumaric acid & 0.9972 & 0.015 & 0.045 & 1.4 & 1.6 \\
Rosmarinic acid & 0.9995 & 0.011 & 0.034 & 0.5 & 2.7 \\
Ellagic acid & 0.9981 & 0.175 & 0.531 & 0.3 & 1.4 \\
3,4-dihydroxybenzoic acid & 0.9997 & 0.011 & 0.033 & 0.9 & 2.5 \\
Abscisic acid & 0.9996 & 0.028 & 0.084 & 0.5 & 1.7 \\
Myricetin & 0.9999 & 0.218 & 0.662 & 1.1 & 1.6 \\
Chrysin & 0.9995 & 0.014 & 0.044 & 2.1 & 2.0 \\
\hline
\end{tabular}

\section{Limit of Detection and Limit of Quantitation}

To estimate the limit of detection (LOD) and limit of quantitation (LOQ), a blank sample (methanol) was spiked three times with each standard at low concentration levels, and the signal-to-noise ratio was determined. The LOD was considered as 3:1, and the LOQ as 10:1. The LOD values were between 0.010 and $0.218 \mu \mathrm{g} / \mathrm{ml}$, while the LOQ values were between 0.029 and $0.662 \mu \mathrm{g} / \mathrm{ml}$ for each standard compound (Table 2).

\section{Statistical Analysis}

Statistical analyses were performed using the Statistica 13.1 software (StatSoft). Means were separated by least significant difference test (LSD) and statistical significance was set at $\alpha=0.05$. Hierarchical cluster analysis (HCA) and principal component analysis (PCA) were used to compare concentrations of identified compounds of honey samples (phenolic acids, flavonoids, and abscisic acid).

\section{Results and Discussion}

\section{Physicochemical Parameters}

The physicochemical parameters studied are moisture content, $\mathrm{pH}$, and electrical conductivity. The mean values for each physicochemical parameter are presented in Table 1 . The honey moisture content is one of the most important parameters, and its level should not exceed $20 \%$ in accordance with international regulations of quality (Codex Alimentarius 2001) and the Polish Quality Standard (PN$88 /$ A-77626 1998). The levels higher than $20 \%$ are indicative of the acceleration of fermentation processes during storage (Semkiw et al. 2008, Muruke 2014).
The electrical conductivity values in honey samples are in the range of $0.38-1.58 \mathrm{mS} / \mathrm{cm}$. The highest values $(1.16-$ $1.58 \mathrm{mS} / \mathrm{cm}$ ) obtained for pine honeydew honeys are according to Polish Quality Standard and Codex Alimentarius. The significant difference in electrical conductivity is used to differentiate honeydew honeys from nectar honeys.

Generally, honeys have an acidic character. In the case of honey samples analyzed, the $\mathrm{pH}$ ranged between 3.92 and 4.62. These values were similar to those previously reported in other honeys (Fechner et al. 2016, Chuttong et al. 2016). The highest levels of $\mathrm{pH}$ were observed in the case of honeydew honeys while willow had the lowest level of $\mathrm{pH}$. Some authors suggest that $\mathrm{pH}$ has an effect on the extraction and storage of honey, but not on the botanical origin (Oroian and Sorina 2017).

\section{HPTLC Fingerprints of Honey}

Each of the received HPTLC images was evaluated to assess whether they provided useful information for the discrimination of the honey brands. The HPTLC chromatograms showed differences in the chemical composition of the honey samples with different origins. All of the honey extracts were enriched in phenolic compounds, and their patterns were dominated by light blue, blue, navy blue, yellow, and black bands. The HPTLC fingerprint analysis allowed the differentiation of honey samples depending on the fluorescent color of the phenolic compounds and showed that these compounds were present in some honey samples in varying amounts (Table 3).

The fingerprint of certain honeys exhibits a unique profile of bands that correspond to the phenolics and can be considered unique because they visually differ from each other. Fig. 1 shows the typical HPTLC fingerprints of willow honey samples (W1-W3). Major bands at $R_{f}$ of 0.03 (light blue), 0.05 (light blue), 0.13 (blue), 0.40 (yellow), 0.45 (blue), 0.50 (blue), 0.59 (navy blue), and 0.61 (yellow) under $366 \mathrm{~nm}$ irradiation can be seen. Under $254 \mathrm{~nm}$, the most prominent 
Table 3 Average $\mathrm{R}_{\mathrm{f}}$ values of identified standards separated by HPTLC and their respective colors

\begin{tabular}{|c|c|c|c|c|}
\hline \multirow[t]{2}{*}{ Standard } & \multirow[t]{2}{*}{ Honey samples } & \multicolumn{2}{|l|}{ Fluorescence } & \multirow[t]{2}{*}{$\mathrm{R}_{\mathrm{f}}$} \\
\hline & & $\lambda=254 \mathrm{~nm}$ & $\lambda=366 \mathrm{~nm}$ & \\
\hline Chlorogenic acid & W1-W3, H1-H2, PH1-PH6 & Light blue & Blue & 0.03 \\
\hline Gallic acid & PH1-PH6 & Navy blue & Black & 0.29 \\
\hline Caffeic acid & W1-W3, H1-H2, PH1-PH6 & Light blue & Light blue & 0.41 \\
\hline Ferulic acid & W1-W3, PH1-PH6 & Blue & Navy blue & 0.51 \\
\hline p-Coumaric acid & B1-B4, H1-H2, M1-M2 & Navy blue & Navy blue & 0.58 \\
\hline Rosmarinic acid & M1-M2 & Blue & Blue & 0.28 \\
\hline Ellagic acid & M1-M2 & Navy blue & Navy blue & 0.44 \\
\hline 3,4-Dihydroxybenzoic acid & W1-W3 & Green & Blue & 0.50 \\
\hline Abscisic acid & W1-W3, H1-H2 & Black & - & 0.49 \\
\hline Myricetin & $\begin{array}{l}\text { W1-W3, H1-H2, B1-B4, } \\
\text { PH1-PH6, M1-M2 }\end{array}$ & Green & Yellow & 0.39 \\
\hline Chrysin & $\mathrm{H} 1-\mathrm{H} 2$ & - & Yellow & 0.66 \\
\hline
\end{tabular}

bands are at $R_{f}$ of 0.03 (light blue), 0.05 (light blue), 0.13 (blue), 0.40 (green), 0.45 (blue), 0.50 (green), 0.52 (black), 0.59 (blue), and 0.61 (green) (Fig. 2). After derivatization with $\mathrm{NEU}$ and $1 \%$ methanolic $\mathrm{AlCl}_{3}$ spraying reagent, the bands at $R_{f} 0.40$ and 0.61 are intensely yellow, and the appearance of new bands at 0.70 (orange) and 0.71 (yellow) can be observed. It is also seen that the phenolic compounds in willow honey change their composition over time from the harvest period. The track profiles for samples of differing harvesting years exhibited the same band profiles, but the older samples (W1 and W2) exhibit bands with a lower intensity $\left(R_{f}=0.40\right)$. We have identified the following phenolic compounds (based on the similarity of the $R_{f}$ and the color of the bands): $R_{f}=0.03$ chlorogenic acid, $R_{f}=0.40$ myricetin, $R_{f}=0.45$ caffeic acid, $R_{f}=0.50$ 3,4-dihydroxybenzoic acid, $R_{f}=0.52$ abscisic acid, and $R_{f}=0.59$ ferulic acid, and the most intense signals were derived from chlorogenic acid (light blue), myricetin (yellow), and abscisic acid (black).

The heather honey samples (H1-H2) exhibit notable bands at $R_{f} 0.03$ (light blue), 0.07 (light blue), 0.09 (yellow), 0.13 (yellow), 0.16 (blue), 0.19 (yellow), 0.29 (yellow), 0.34 (yellow), 0.37 (yellow), 0.40 (blue), 0.47 (blue), 0.55 (dark blue),

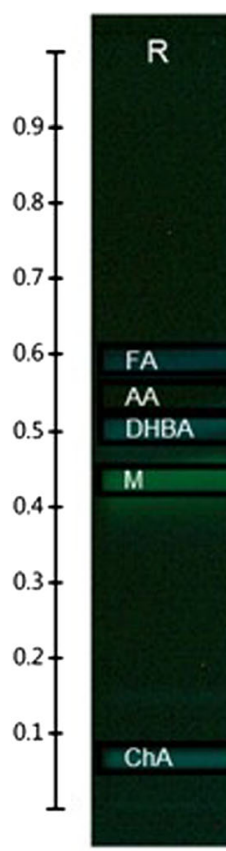

1

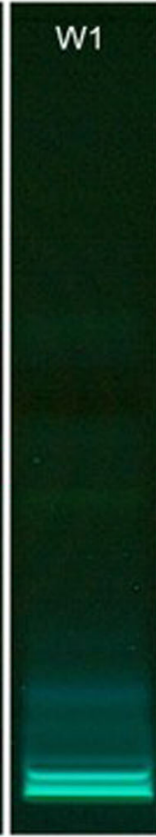

2

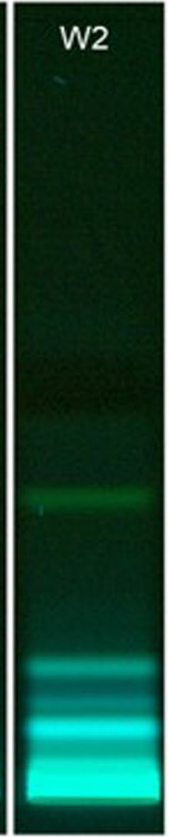

3

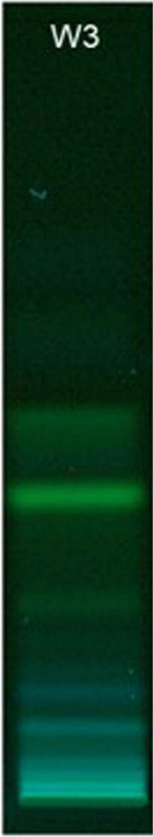

4

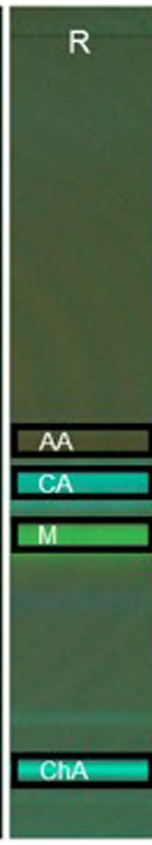

5

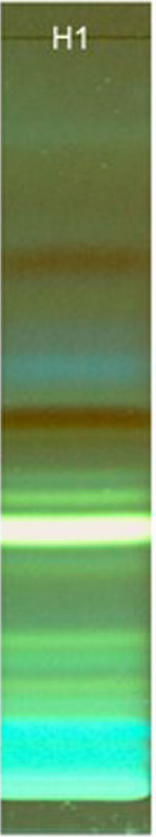

6

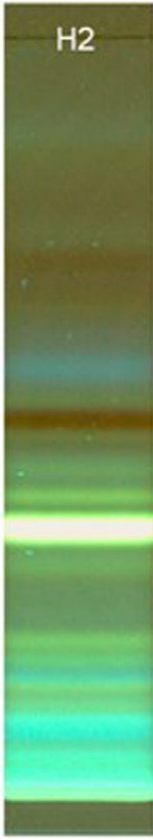

7

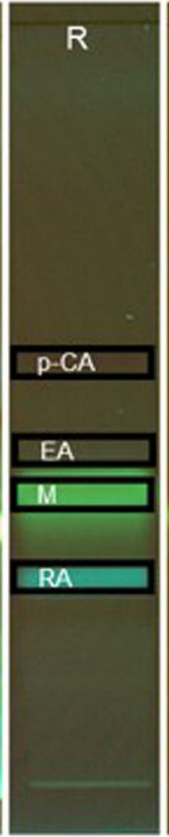

8

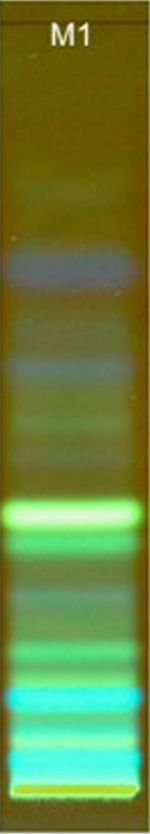

9

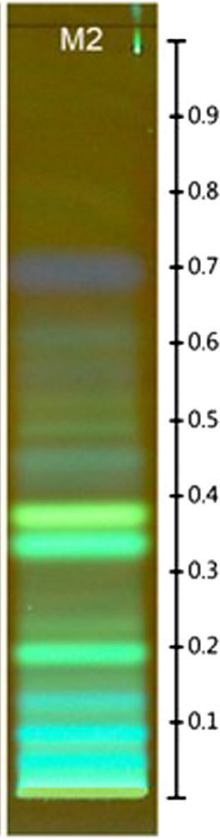

10

Fig. 1 HPTLC chromatogram at $366 \mathrm{~nm}$, W1-W3 willow honey samples, H1-H2 heather honey samples, M1-M2- manuka honey samples, R references, FA ferulic acid, DHBA 3,4-dihydroksybenzoic

acid, M myricetin, ChA chlorogenic acid, Ch chrysin, $p$-CA $p$-coumaric acid, CA caffeic acid, EA ellagic acid, RA rosmarinic acid 


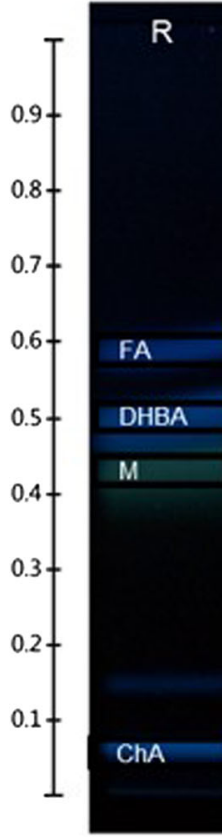

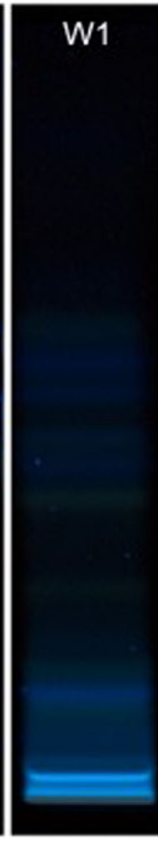

2

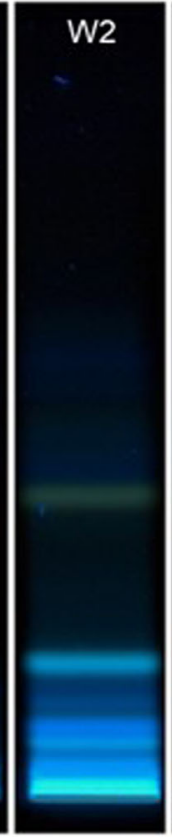

3

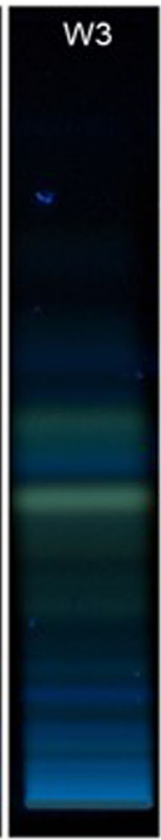

4

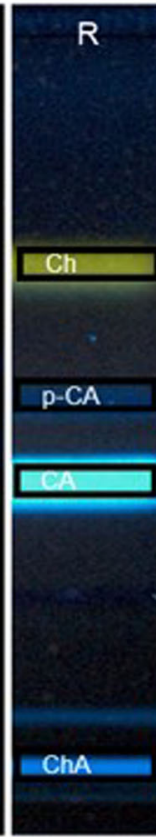

5

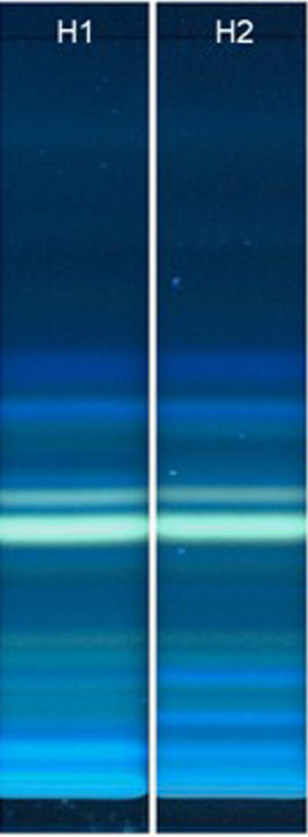

7

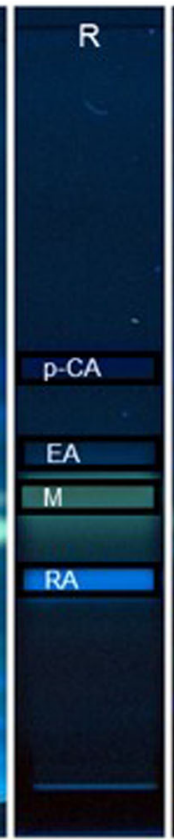

8

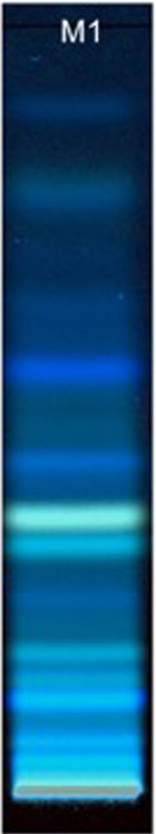

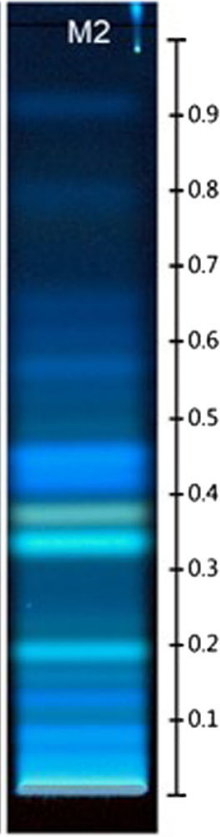

10

Fig. 2 HPTLC chromatogram at $254 \mathrm{~nm}$. W1-W3 willow honey samples, H1-H2 heater honey samples, M1-M2 manuka honey samples, $\mathrm{R}$ references, FA ferulic acid, DHBA 3,4-dihydroksybenzoic acid, M

0.58 (navy blue), and 0.66 (yellow) under $366 \mathrm{~nm}$ (Fig. 1). Additionally, visualization with $254 \mathrm{~nm}$ results in spots of $R_{f}$ of 0.03 (light blue), 0.07 (light blue), 0.09 (yellow), 0.13 (yellow), 0.16 (yellow), 0.19 (yellow), 0.29 (yellow), 0.34 (intense yellow), 0.37 (yellow), 0.40 (blue), 0.47 (black), 0.55 (blue), 0.58 (blue), and 0.66 (yellow) (Fig. 2). Based on the color reactions of polyphenols before and after the derivatization, the following components were identified: $R_{f}=0.03$ chlorogenic acid, $R_{f}=0.34$ myricetin, $R_{f}=0.40$ caffeic acid, $R_{f}=0.47$ abscisic acid, $R_{f}=0.58 p$-coumaric acid and $R_{f}=$ 0.66 chrysin. It is noteworthy that the chromatogram was dominated by the intense yellow and black bands that were identified as myricetin and abscisic acid, respectively. In addition, the heather honey profiles at $366 \mathrm{~nm}$ irradiation showed great similarity in the phenolic signals, which indicated that all of the samples belong to the same type of honey (Fig. 5). The strongest signal was observed at $R_{f}=0.34$, which originated from myricetin.

The buckwheat honey samples (B1-B4) exhibited bands of interest at $R_{f}$ of 0.03 (light blue), 0.05 (dark blue), 0.08 (blue), 0.14 (dark blue), 0.18 (blue), 0.25 (yellow), 0.36 (navy blue), 0.38 (yellow), 0.42 (dark blue), 0.46 (dark blue), 0.54 (dark blue), 0.57 (navy blue), and 0.68 (dark blue) under $366 \mathrm{~nm}$ (Fig. 3). UV irradiation at $254 \mathrm{~nm}$, there are bands at $\mathrm{R}_{\mathrm{f}}$ of 0.03 (light blue), 0.05 (dark blue), 0.08 (white), 0.10 (dark blue), 0.14 (dark blue), 0.25 (yellow), 0.36 (light blue), 0.38 (yellow), 0.42 (dark blue), 0.46 (dark blue), 0.54 (dark blue), 0.57 (navy blue), and 0.68 (dark blue) (Fig. 4). Two phenolic myricetin, ChA chlorogenic acid, $p$-CA $p$-coumaric acid, CA caffeic acid, EA ellagic acid, RA rosmarinic acid, AA abscisic acid

compounds were identified here: $R_{f}=0.38$ myricetin and $R_{f}=0.57 p$-coumaric acid (Fig. 4).

Figure 3 shows the various pine honeydew extract chromatograms. Visualization at $366 \mathrm{~nm}$ unveils notable bands at $R_{f}$ values of 0.03 (light blue), 0.05 (blue), 0.11 (dark blue), 0.13 (dark blue), 0.30 (black), 0.34 (orange), 0.38 (yellow), 0.39 (blue), 0.46 (black), 0.51 (navy blue), and 0.66 (dark blue). The derivatized samples (NEU reagent) have a pronounced band at $R_{f}=0.54$ (yellow). UV irradiation with $254 \mathrm{~nm}$, the samples contain additional spots at $R_{f}=0.08$ (light blue), 0.17 (light blue), 0.43 (yellow), 0.51 (dark blue), and 0.54 (yellow) (Fig. 4). HPTLC led to the identification of four phenolic acids, chlorogenic $\left(R_{f}=0.03\right)$, gallic $\left(R_{f}=0.30\right)$, caffeic $\left(R_{f}=0.39\right)$ and ferulic $\left(R_{f}=0.51\right)$, and one flavonoid, myricetin $\left(R_{f}=\right.$ 0.38 ). As observed in Fig. 5, various pine honeydew honey sample profiles under $366 \mathrm{~nm}$ display very similar profiles with key peaks at $\mathrm{R}_{\mathrm{f}}=0.03,0.13,0.46$, and 0.51 .

Figures 1 and 2 illustrate the chromatograms of manuka honey obtained from different suppliers and locations with varying ages (Table 1). Still, the manuka honey extract under $366 \mathrm{~nm}$ irradiation exhibits a very similar fingerprint with key peaks at $R_{f}=0.03$ (light blue), 0.06 (light blue), 0.10 (light blue), 0.14 (blue), 0.21 (light blue), 0.24 (white), 0.34 (light blue), 0.38 (yellow), 0.44 (navy blue), 0.47 (dark blue), 0.57 (dark blue), 0.69 (navy blue), 0.78 (dark blue), 0.89 (dark blue), and 0.28 (dark blue), which are characteristic for only the M1 honey samples (Fig. 1). Under $254 \mathrm{~nm}$, there are prominent additional bands at $R_{f}=0.24$ (yellow), 0.47 (dark 


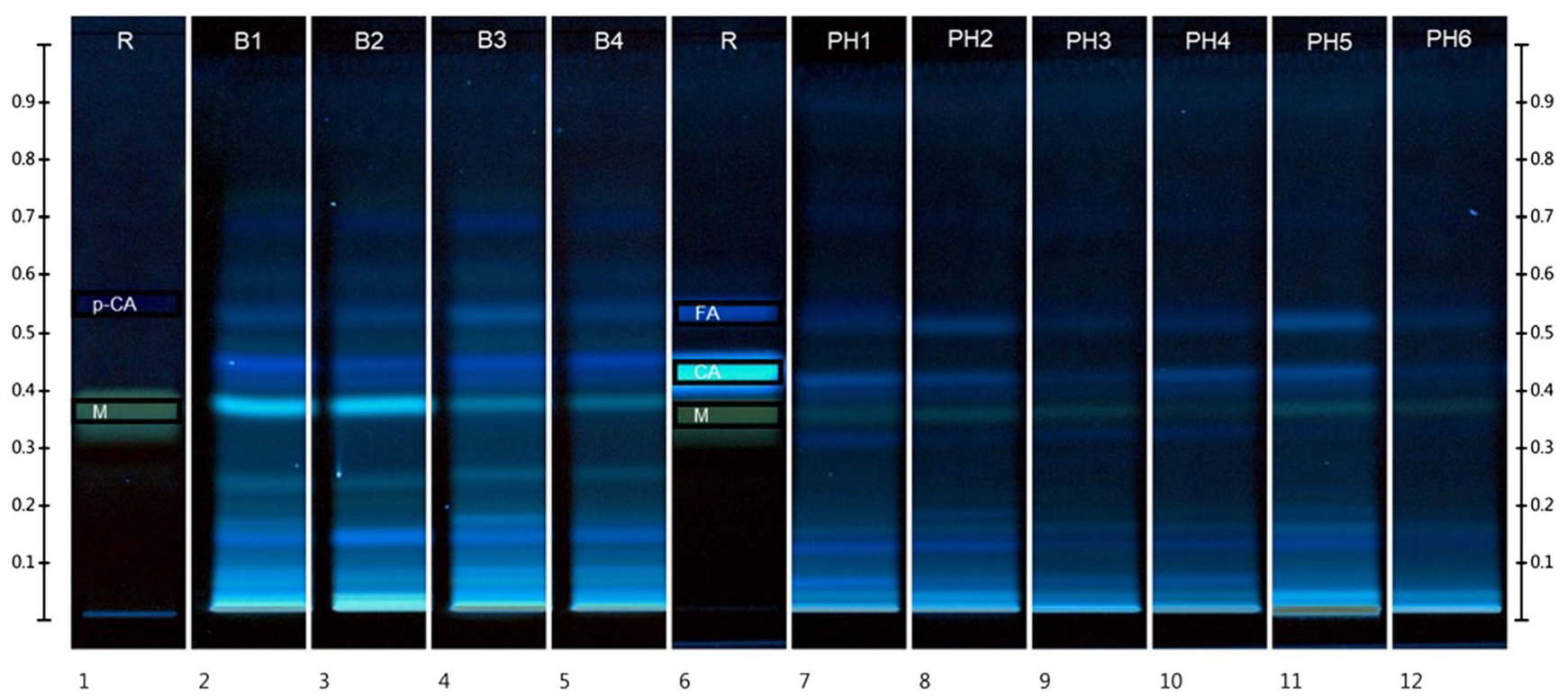

Fig. 3 HPTLC chromatogram at $366 \mathrm{~nm}$. B1-B4 buckwheat honey samples, PH1-PH6 pine honeydew honey samples, R references, $p$-CA $p$-coumaric acid, M myricetin, FA ferulic acid, CA caffeic acid

blue), 0.50 (light blue), and 0.62 (light blue), while bands at $R_{f}=0.78$ and $R_{f}=0.89$ are not visible (Fig. 2). Manuka samples contained rosmarinic acid $\left(R_{f}=0.28\right)$, ellagic acid $\left(R_{f}=\right.$ $0.44), p$-coumaric acid $\left(R_{f}=0.57\right)$, and myricetin $\left(R_{f}=0.38\right)$. As is illustrated in Fig. 5, despite very similar profiles, differences between the samples M1 and M2 are observed. Under $366 \mathrm{~nm}$ irradiation, a loss in the band intensity at $0.10,0.21$, 0.34 , and 0.47 and an intense band appearance at $0.14,0.38$, and 0.57 in sample M1 are observed, which is in contrast to the M2 sample. These insignificant differences are most likely the result of the geographical origin of these honey samples.

\section{Identification of Phenolic Compounds by HPLC}

Eleven polyphenolics were identified in honey samples by HPTLC, and the obtained results were further confirmed by HPLC analysis. HPLC was used for the quantification of phenolic compounds in all honey samples. Initially, the validation parameters, such as linearity, precision, limit of detection, and quantification of the developed method (HPLC-DAD), were established (Table 2). The concentrations of all of the identified phenolic compounds in the honey samples are given in Table 4. The HPLC analysis of willow honey (W1-W3)

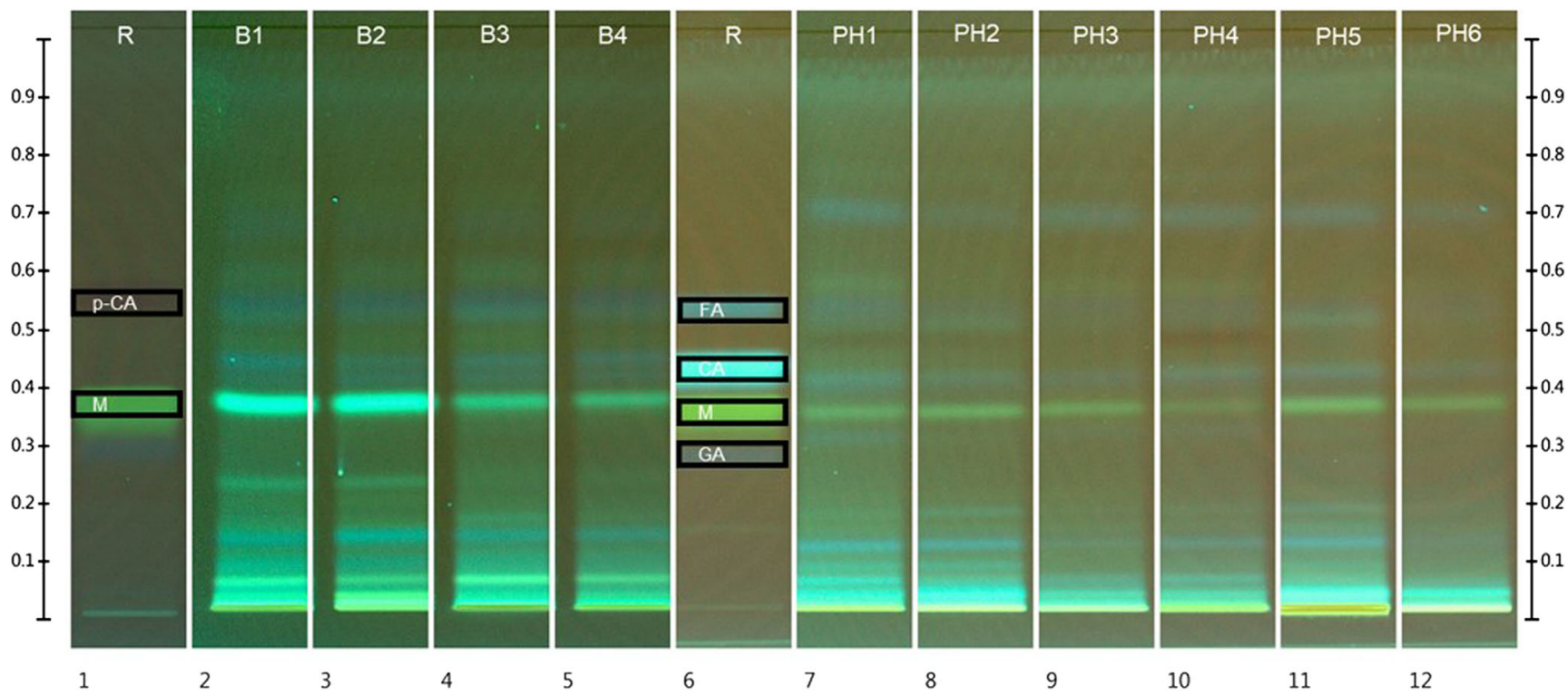

Fig. 4 HPTLC chromatogram at $254 \mathrm{~nm}$. B1-B4 buckwheat honey samples, PH1-PH6 pine honeydew honey samples, R references, $p$-CA $p$-coumaric acid, M myricetin, FA ferulic acid, CA caffeic acid, GA gallic acid 


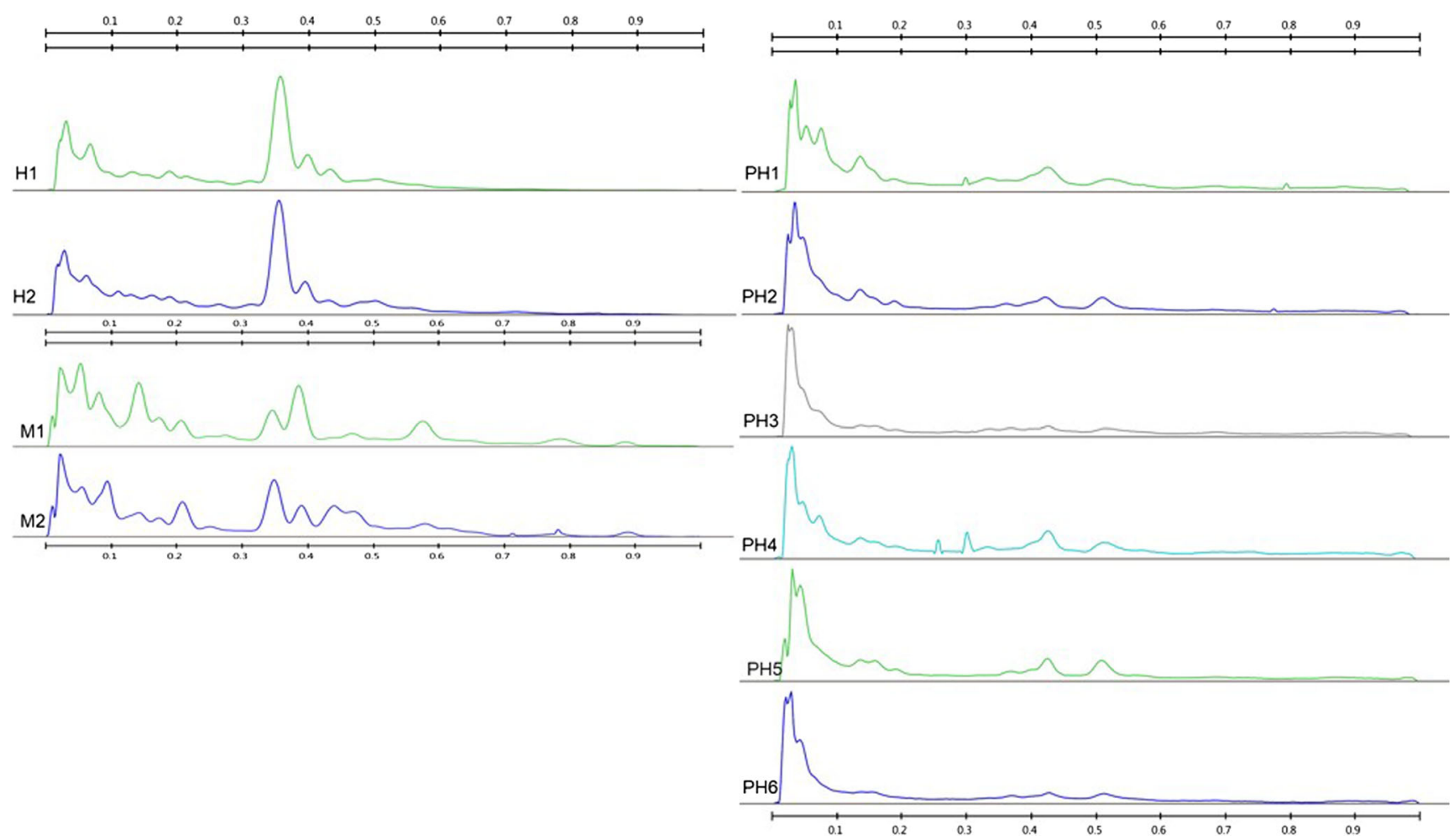

Fig. 5 HPTLC honey extract profiles at $366 \mathrm{~nm}$. H1-H2 heater honey samples, PH1-PH6 pine honeydew honey samples, M1-M2 manuka honey samples

confirmed the presence of chlorogenic acid, myricetin, caffeic acid, 3,4-dihydroxybenzoic acid, abscisic acid, and ferulic acid. Their content varied in the range from 5.276 to $7.643 \mathrm{mg}$ per $100 \mathrm{~g}$ of honey. Abscisic acid was found in high quantities. In relation to the literature data, abscisic acid was found in Polish (Jerkovic et al. 2014) and Croatian willow honey (Tuberoso et al. 2011) in concentrations of 25.8-35.6 and $31.7-98.2 \mathrm{mg} / \mathrm{kg}$, respectively. A similar dependence between the composition of the willow honey samples and the harvest year was observed by both HPTLC and HPLC. The highest amounts of caffeic acid, ferulic acid, and myricetin were found in the W3 sample collected in 2016 compared with the W1 and W2 samples that were collected in 2015. The level of these compounds gradually decreased with the age of the honey (caffeic acid content decreased to $60 \%$, ferulic acid to $81 \%$, and myricetin to $73 \%$ ). In the case of other identified phenolic compounds, no significant differences in their concentrations were found.

The heather honey exhibited the highest level of abscisic acid (8.959-11.772 mg/100 g of honey) and myricetin (0.639-0.861 mg/100 g of honey). Similar content values of these compounds were observed by Jasicka-Misiak et al. 2012 (abscisic acid 8.272-12.061 mg/100 g of honey; myricetin $0.161-1.485 \mathrm{mg} / 100 \mathrm{~g}$ of honey). Additionally, a high level of abscisic acid (2.5-16.66 mg/100 g honey) in heather honey was identified by Ferreres et al. 1996 who considered this compound as a potential marker for this honey.
Myricetin and $p$-coumaric acid dominated in buckwheat honey. The content of these phenolics ranged from 0.243 to 0.606 and 0.267 to $0.489 \mathrm{mg} / 100 \mathrm{~g}$ of honey, respectively. A similar phenolic profile was found by Jasicka-Misiak et al. 2012 with a myricetin level of $0.082-1.907 \mathrm{mg} / 100 \mathrm{~g}$ of honey and $p$ coumaric acid of $0.026-4.551 \mathrm{mg} / 100 \mathrm{~g}$ of honey. Moreover, according to Pasini et al. (2013), the quantified levels of $p$ coumaric acid in buckwheat honey were 0.058-1.297 mg/ $100 \mathrm{~g}$ of honey. In another study, carried out by Karabagias et al. (2014), it was reported that myrycetin and chrysin were determined in thyme honeys. Myrycetin ranged from 0.74 to $244.67 \mathrm{mg} / \mathrm{kg}$ and chrysin ranged from 0.01 to $5.60 \mathrm{mg} / \mathrm{kg}$.

The most prominent components of pine honeydew honey were chlorogenic acid $(0.179-0.824 \mathrm{mg} / 100 \mathrm{~g})$, gallic acid $(1.315-4.793 \mathrm{mg} / 100 \mathrm{~g})$, caffeic acid $(0.049-0.337 \mathrm{mg} /$ $100 \mathrm{~g})$, ferulic acid $(0.039-0.620 \mathrm{mg} / 100 \mathrm{~g})$, and myricetin $(0.179-0.842 \mathrm{mg} / 100 \mathrm{~g})$. Gallic acid was found to be in the largest amounts, whereas caffeic acid, chlorogenic acid, ferulic acid, and myricetin were at lower levels. The level of phenolic compounds in honeydew honey was higher than those obtained in other studies (Oroian and Sorina 2017; Socha et al. 2011), but the values obtained may vary depending on the type of honeydew used by bees for production of certain honey.

The chromatography data confirmed the already described presence (Yao et al. 2003) of rosmarinic, ellagic, $p$-coumaric acids, and myricetin in manuka honey. The Leptospermum honey, including Australian and New Zealand manuka honey, 


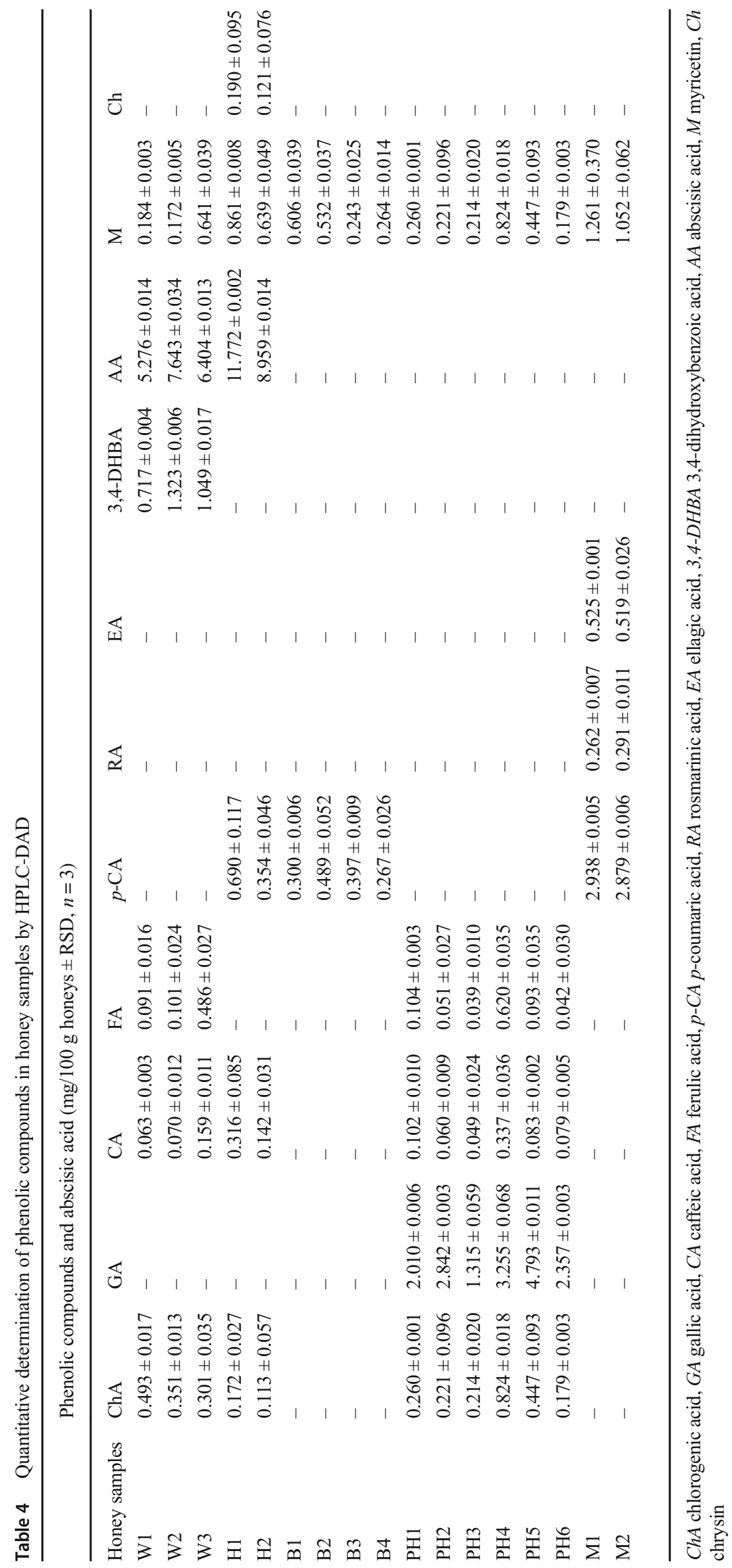



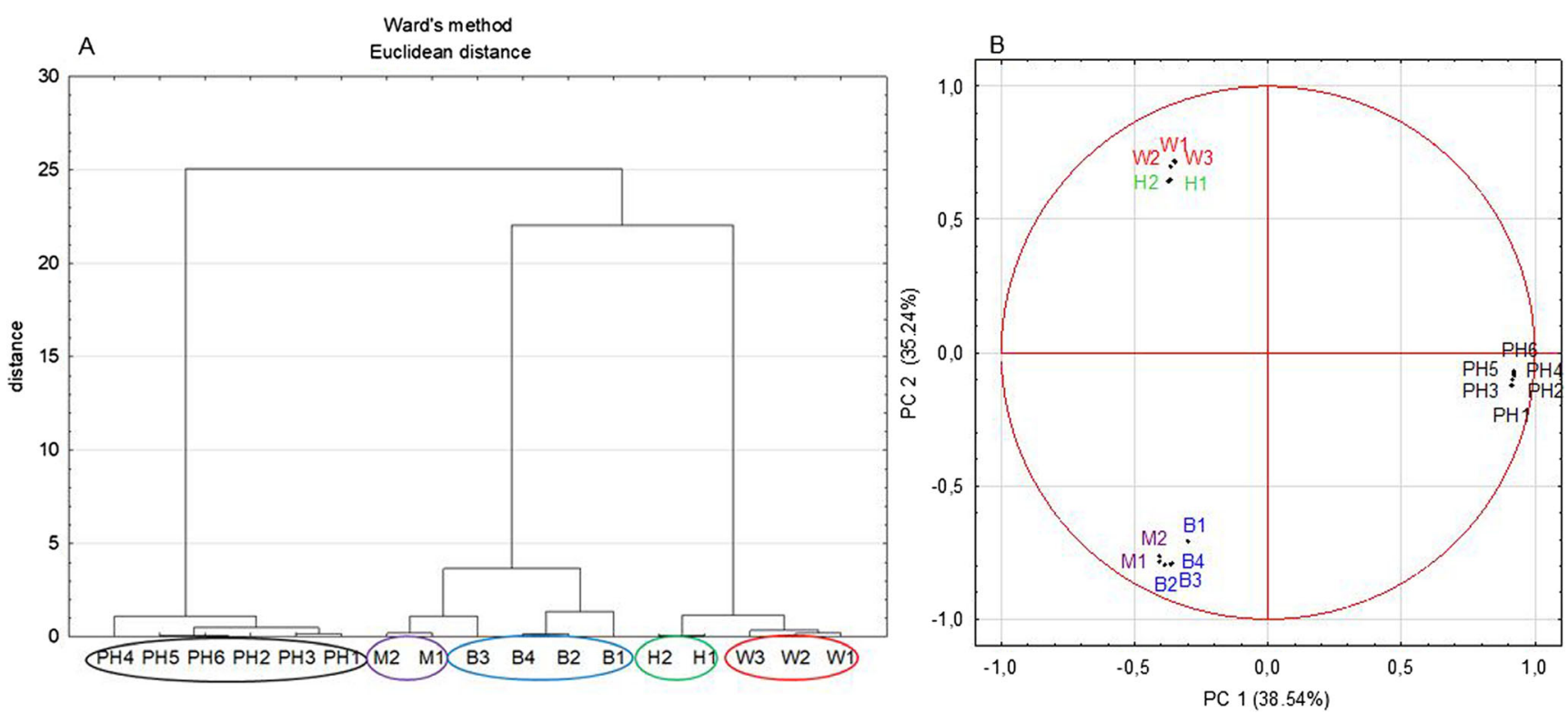

Fig. 6 Hierarchical cluster analysis dendrograms (a) and principal component analysis (b) of the tested honey samples. W1-W3 willow, H1-H2 heather, B1-B4 buckwheat, Ph1-Ph6 pine honeydew, M1-M2 manuka honey

presented standard phenolic profiles with only slight differences between the varieties. Thus, the quantified levels of $p$-coumaric, rosmarinic, and ellagic acid in Australian manuka honey were slightly higher than those in New Zealand manuka honey, which additionally exhibited lower values of myricetin.

\section{Multivariate Data Analysis}

This study aimed to determine the compounds such as phenolic acids, flavonoids, and abscisic acid which could be very useful when classifying honeys according to their variety. HCA and PCA were used to establish the relationship between the chemical composition of honeys and their floral origin. The results of HCA are presented as dendrograms in Fig. 6 a. All honey samples were classified into three clusters (A, B, and C). The cluster A contains of pine honeydew samples. This group clearly differed from other tested honey. The samples manuka and buckwheat honeys formed cluster B, which showed weak similarity with samples heather and willow honeys, distributed cluster C. Among the samples in the third group, willow honey samples clearly differed from heather honey samples. Moreover, this tendency was repeated in the PCA classification. The components explained $74.95 \%$ of the total variance (PC1 $39.16 \%$ and $\mathrm{PC} 235.79 \%$ ). Figure $6 \mathrm{~b}$ show the clustering of honey samples into three main groups, such as willow and heather (first), buckwheat and manuka (second), and pine honeydew honeys (third). Principal component analysis clearly differentiates pine honeydew located on the right side from other honey samples. Willow and heather honeys samples are grouped into one cluster. This is probably due to the fact that these samples were characterized by large amounts of abscisic acid. The cluster buckwheat and manuka honey samples partly overlap, but a certain separation can be observed. In both honeys, $p$-coumaric acid and myricetin were identified, but the manuka honeys were additionally characterized by the occurrence of rosmarinic acid and ellagic acid. Comparing HCA and PCA technique obtained results that exhibited possible relationship to botanical origin of honey samples. Accordingly to the phenolic properties, honeydew honey samples were perfectly classified, while manuka and buckwheat or willow and heather samples partly overlap. Additionally, the similarity of honeys with large percentage of pollen type (W2-W3, B1) and with pollen of less than 45\% (W1, B2-B4) was observed, which shows that the slightly lower value of pollen in honeys is not different from honey with dominance of one type pollen.

\section{Conclusions}

The low instrumentation price, short analysis time, wide range of adsorbents and solvents, small sample requirements for analysis, good precision, and accuracy make HPTLC a method of choice for the differentiation of the botanical origin of honey samples. In the present study, the HPTLC fingerprint analysis allowed the identification of the type of the following samples of honey: willow, buckwheat, heather, pine honeydew, and manuka. This showed that on the basis of the similarities and differences in the observed bands, it was possible to determine the botanical origin of certain honey. Additionally, 11 phenolic compounds were identified, and their presence was independently confirmed by an HPLC analysis. HPTLC has been proven to be a good method for the rapid separation of honey components and well suited for the identification of phenolic compounds present in honey samples. It should also be emphasized 
that the HPTLC analysis of honey might assist in developing HPTLC-supported quality control tools and might improve consumer confidence and support to the product.

This work was supported by The National Science Centre of Poland in the frame of Grant No. 2014/15/B/N29/02182.

Funding This work was supported by The National Science Centre of Poland in the frame of Grant No. 2014/15/B/NZ9/02182.

\section{Compliance with Ethical Standards}

Conflict of Interest Natalia Stanek declares that she has no conflict of interest. Izabela Jasicka-Misiak declares that she has no conflict of interest.

Ethical Approval This article does not contain any studies with human participants performed by any of the authors.

\section{Informed Consent Not applicable.}

Open Access This article is distributed under the terms of the Creative Commons Attribution 4.0 International License (http:// creativecommons.org/licenses/by/4.0/), which permits unrestricted use, distribution, and reproduction in any medium, provided you give appropriate credit to the original author(s) and the source, provide a link to the Creative Commons license, and indicate if changes were made.

\section{References}

Badjah Hadj Ahmed AY, Wabaidur SM, Siddiqui MR, Alothman ZA, Obeid MS, Khan MR, Tamrah AL (2016) Simultaneous determination of twenty-five polyphenols in multifloral and cactus honeys using solid-phase extraction and high-performance liquid chromatography with photodiode array detection. Eur Food Res Technol 242:943-952

Bogdanov S, Ruoff K, Oddo L (2004) Physico-chemical methods for the characterisation of unifloral honeys: a review. Appidolgie 35:S4-S17

Chuttong B, Chanbang Y, Sringarm K, Burgett M (2016) Physicochemicals profiles of singles bee (Apidae: Meliponini) honey from South East Asia (Thailand). Food Chem 192:149-155

Ciulu M, Spano N, Pilo MI, Sanna G (2016) Recent advances in the analysis of phenolic compounds in unifloral honeys. Molecules 21:451-482

Codex Alimentarius (2001) revised codex standards for honey. Codex standard 12-1981

Dinca RF, Ionete RE, Popescu R, Costinel D, Radu GL (2015) Geographical and botanical origin of Romanian honey using complex stable isotope data and chemometrics. Food Anal Methods 8:401-412

Fechner DC, Moresi AL, Diaz JDR, Pellerano RG, Vazquez FA (2016) Multivariate classification of honeys from Corrientes (Argentina) according to geographical origin based on physicochemical properties. Food Biosci 15:49-54

Ferreres F, Andrade P, Tomas-Barberan FA (1996) Natural occurrence of abscisic acid in heather honey and floral nectar. J Agric Food Chem 44:2053-2056

Głowniak K, Skalicka K, Ludwiczuk A, Jop K (2005) Phenolic compounds in the flowers of Lavatera trimestris L. (Malvaceae). J Planar Chromatogr 18:264-268

Jasicka-Misiak I, Poliwoda A, Dereń M, Kafarski P (2012) Phenolic compounds and abscisic acid as potential markers for the floral origin of two Polish unifloral honeys. Food Chem 131:1149-1156
Jasicka-Misiak I, Makowicz E, Stanek N (2017) Polish yellow sweet clover (Melilotus officinalis L.) honey, chromatographic fingerprints and chemical markers. Molecules 22:138

Jerkovic I, Kuś PM, Tuberoso CIG, Šarolic M (2014) Phytochemical and physical-chemical analysis of Polish willow (Salix spp.) honey: identification of the marker compounds. Food Chem 145:8-14

Karabagias IK, Vavoura MV, Badeka A, Kontakos S, Kontaminas MG (2014) Differentiation of Greek thyme honeys according to geographical origin based on the combination of phenolic compounds and conventional quality parameters using chemometrics. Food Anal Methods 7:2113-2121

Kasprzyk I, Depciuch J, Grabek-Lejko D, Parlinska-Wojtan M (2018) FTIRATR spectroscopy of pollen and honey as tool for unifloral honey authentication. The case study of rape honey. Food Control 84:33-40

Kečkeš S, Gašić U, Veličković TĆ, Milojković-Opsenica D, Natić M, Tešić Ž (2013) The determination of phenolic profiles of Serbian unifloral honeys using ultra-high-performance liquid chromatography/high resolution accurate mass spectrometry. Food Chem 138:32-40

Kuś PM, Congiu F, Teper D, Sroka Z, Jerković I, Tuberoso CIG (2014) Antoxidant activity, color chracterisitics, total phenol content and general HPLC fingerprints of six Polish unifloral honey types. LWT Food Sci Technol 55:124-130

Locher C, Neumann J, Sostaric T (2017) Authentication of honeys of different floral origin via high-performance thin-layer chromatographic fingerprinting. J Planar Chromatogr 30:57-62

Milojković Opsenica D, Ristivojević P, Trifković J, Vovk I, Lušić D, Tešić Ž (2016) TLC fingerprinting and pattern recognition methods in the assessment of authenticity of poplar-type propolis. J Chromatogr Sci 54:1077-1083

Muruke MH (2014) Assessment of quality of Tanzanian honey based on physicochemical properties. Food Sci 33:61-72

Oroian M, Sorina R (2017) Honey authentication based on physicochemical parameters and phenolic compounds. Comp Electron Agric 138: $148-156$

Pascual-Maté A, Osés SM, Fernández-Muiño MA, Sancho MT (2017) Analysis of polyphenols in honey: extraction, separation, and quantification procedures. J Sep Purif 16:1-17

Pasini F, Gardini S, Marcazzan GL, Caboni MF (2013) Buckwheat honeys: screening of composition and properties. Food Chem 141: 2802-2811

PN-88/A-77626 (1998) Miód pszczeli. [Polish Quality Standard "Honey"] Dziennik Norm i Miar nr 8, Wydawnictwo Normalizacyjne Alfa

Pyrzyńska K, Biesaga M (2009) Analysis of phenolic acids and flavonoids in honey. Trends Anal Chem 28:893-902

Ristivojević P, Trifković J, Vovk I, Milojković-Opsenica D (2017) Comparative study of different approaches for multivariate image analysis in HPTLC fingerprinting of natural products such as plant resin. Talanta 162:72-79

Semkiw P, Skowronek W, Skubida P (2008) Changes in water content of honey during ripening under controlled condition. J Apic Sci 52:57-63

Socha R, Juszczak L, Pietrzyk S, Gałkowska D, Fortuna T, Witczak T (2011) Phenolic profile and antioxidant properties of Polish honeys. Int J Food Sci Technol 46:528-534

Tuberoso CIG, Jerkovic I, Bifulcoa E, Marijanovic Z (2011) Biodiversity of Salix spp. honeydew and nectar honeys determined by RP-HPLC and evaluation of their antioxidant capacity. Chem Biodivers 8:872-879

Waterman PG (1994) Methods in Ecology. Analysis of Phenolic Plant Metabolites. Oxford, pp 151-154

Yao L, Datta N, Tomas-Barberan FA, Ferreres F, Martos I, Singanusong R (2003) Flavonoids, phenolic acids and abscisic acid in Australian and New Zealand Leptospermum honeys. Food Chem 81:159-168

Żyszka B, Anioł M, Lipok J (2017) Highly effective, region specific reduction of chalcone by cyanobacteria leads to the formation of dihydrochalcone: two steps towards natural sweetness. Microb Cell Fact 16:136-151 